\title{
Fundamental Group of Rough Topological Spaces
}

\author{
Laaro Abdullateef \\ Department of Mathematical Sciences, Bayero University Kano, Kano, Nigeria \\ e-mail: laaroabdullateef@gmail.com
}

\begin{abstract}
In this paper we study and define the concept of the fundamental group of rough topological spaces (RTSs), which deeply depends on the concepts of rough sets (RSs) and rough topology (RT). Working towards this stated objective, we define the concept of rough path $(\mathrm{RPt})$ which gives room for the introduction of rough loop (RL). We also define the concepts of rough homotopy $(\mathrm{RH})$ and later shows that it is indeed an equivalence relation. We introduce the fundamental group of rough topological spaces by showing that all the group axioms satisfied. Also, this paper establish the fact that most of the results in fundamental group of ordinary topological spaces are also hold for the fundamental group of rough topological spaces.
\end{abstract}

\section{Introduction}

Due to the applications of classical method in solving various types of inexact or uncertainties problems in economics, engineering and environment, several theories which include: the theory of probability, the theory of fuzzy sets, theory of rough sets and the interval mathematics are introduced as mathematical tools for dealing with these uncertainties [8].

Rough sets was proposed by Pawlak [21] as a useful tool to deal with uncertainty and incomplete information and it has been approved to be effective approach to intelligent systems characterized by insufficient and incomplete information [26]. Since then rough sets and its applications have attracted the interest of researchers in many fields [10]. This includes, the generalized multi-fuzzy rough sets and the induced topology [9], rough set theory for topological spaces [8], generalized rough sets based on neighborhood systems Received: June 29, 2020; Revised: August 1, 2020, Accepted: August 5, 2020 2010 Mathematics Subject Classification: 03E72, 54A05, 54A40.

Keywords and phrases: rough sets (RSs), rough topological spaces (RTSs), rough path (RPt), rough loop $(\mathrm{RL})$, rough homotopy $(\mathrm{RH})$. 
and topological spaces [17], topological structure of generalized rough sets [22], topological properties of IF approximation spaces [16], topological structures of IVF approximation spaces [10], rough topological spaces [3], fuzzy rough sets and their topological structures [19], generalized intuitionistic fuzzy soft rough set and its application in decision making [12], I-rough topological spaces and some of its special properties $[4,5,6]$.

Considering the fact that in recent years many researchers have considered fundamental group introduced by Poincare [2] in the study of other mathematical approach, which includes the fundamental group as a topological group [9], the fundamental of intuitionistic fuzzy topological spaces and its algebraic properties [7, 15], the fundamental group of quotient spaces [13], the Fuzzy $\mathfrak{S}^{*}$-Fundamental Group of Fuzzy $\mathfrak{J}^{*}$-Structure Spaces [18], monoids in the fundamental groups of the complement of logarithmic free divisors in $C^{3}$ [14]. The applications of fundamental group to the study of an effective approach to intelligent systems, i.e., rough topological spaces will be our priority in this paper.

\section{Preliminaries}

Suppose we are given a nonempty set of objects $U$ called the universe and an equivalence relation called indiscernibility relation $R$ on $U$, then the pair $(U, R)$ is known as the approximation space [23]. Let $X$ be a subset of $U$, in order to characterize $X$ with respect to $R$, two crisp sets will be associate to $X$, called its lower and upper approximations.

Definition 2.1 [23]. The equivalence class of $R$ containing an element $x$ will be denoted by $R(x)$ and is called granules of knowledge generated by $R$, which represents elementary portion of knowledge we are able to perceive due to $R$.

Definition 2.2 [23]. The lower approximation of $X$ denoted by $X_{L}$ is the union of all granules which are entirely included in the set. That is $X_{L}=\{x \mid R(x) \subset X\}$. Therefore lower approximation of a set consists of all elements that surely belong to the set.

Definition 2.3 [23]. The upper approximation of $X$ denoted by $X_{U}$ is the union of all granules which have nonempty intersection with the set. That is $X_{U}=\{x \mid R(x) \cap X \neq \emptyset\}$. Therefore upper approximation of the set constitutes of all elements that possibly belong to the set.

Definition 2.4 [23]. The boundary region of set is the difference between the upper 
and the lower approximation. Intuitively, the boundary region of the set consists of all elements that cannot be classified uniquely to the set or its complement, by employing available knowledge.

Definition 2.5 [23]. A set is said to be a rough set, if it has a non-empty boundary region. If the boundary region is empty, then the set is a crisp or exact set.

In rough set, data model information is stored in a table. Each row (tuples) represents a fact or an object.

Example 2.6.

\begin{tabular}{|c|c|c|c|c|c|}
\hline Object & $\boldsymbol{P}_{\mathbf{1}}$ & $\boldsymbol{P}_{\mathbf{2}}$ & $\boldsymbol{P}_{\mathbf{3}}$ & $\boldsymbol{P}_{\mathbf{4}}$ & $\boldsymbol{P}_{\mathbf{5}}$ \\
\hline $\boldsymbol{o}_{\mathbf{1}}$ & 1 & 2 & 0 & 1 & 1 \\
\hline $\boldsymbol{o}_{\mathbf{2}}$ & 1 & 2 & 0 & 1 & 1 \\
\hline $\boldsymbol{o}_{\mathbf{3}}$ & 2 & 0 & 0 & 1 & 0 \\
\hline $\boldsymbol{o}_{\mathbf{4}}$ & 0 & 0 & 1 & 2 & 1 \\
\hline $\boldsymbol{o}_{\mathbf{5}}$ & 2 & 1 & 0 & 2 & 1 \\
\hline $\boldsymbol{o}_{\mathbf{6}}$ & 0 & 0 & 1 & 2 & 2 \\
\hline $\boldsymbol{o}_{\mathbf{7}}$ & 2 & 0 & 0 & 1 & 0 \\
\hline $\boldsymbol{o}_{\mathbf{8}}$ & 0 & 1 & 2 & 2 & 1 \\
\hline $\boldsymbol{o}_{\mathbf{9}}$ & 2 & 1 & 0 & 2 & 2 \\
\hline $\boldsymbol{o}_{\mathbf{1 0}}$ & 2 & 0 & 0 & 1 & 0 \\
\hline
\end{tabular}

Considering the table above, then

$$
\begin{gathered}
X_{L}=\left\{\left\{o_{1}, o_{2}, o_{5}, o_{8}\right\},\left\{o_{4}, o_{6}, o_{9}\right\}\right\} \\
X_{U}=\left\{\left\{o_{1}, o_{2}, o_{3}, o_{7}, o_{10}, o_{5}, o_{8}\right\},\left\{o_{3}, o_{4}, o_{6}, o_{7}, o_{9}, o_{10}\right\}\right\} \\
X=\left\{\left\{o_{1}, o_{2}, o_{3}, o_{5}, o_{8}\right\},\left\{o_{4}, o_{6}, o_{7}, o_{9}, o_{10}\right\}\right\} \\
P=\left\{P_{1}, P_{2}, P_{3}, P_{4}, P_{5}\right\}
\end{gathered}
$$

where $X$ and $P$ are target set and subset respectively.

Definition 2.7 [23]. Let $A=\left(A_{L}, A_{U}\right)$ and $B=\left(B_{L}, B_{U}\right)$ be any two arbitrary rough sets. Then $A$ is said to be a rough subset of $B$ if and only if $A_{L} \subset B_{L}$ and $A_{U} \subset B_{U}$. 
Definition 2.8 [23]. Let $A=\left(A_{L}, A_{U}\right)$ and $B=\left(B_{L}, B_{U}\right)$ be any two arbitrary rough subsets of $X$. Then the union of rough sets $A$ and $B$ is defined as $A \cup B=\left(A_{L} \cup B_{L}\right.$, $\left.A_{U} \cup B_{U}\right)$ and intersection of $A$ and $B$ is defined as $A \cap B=\left(A_{L} \cap B_{L}, A_{U} \cap B_{U}\right)$.

Definition 2.9 [23]. A set $X$ is said to be exact if $X_{L}=X_{U}$ and it said to be rough if $X_{L} \neq X_{U}$.

Definition 2.10 [3]. Let $X=\left(X_{L}, X_{U}\right)$ be a rough subset of the approximation space $(\omega, R)$. Let $\tau_{L}$ and $\tau_{U}$ be any two topologies which contain only exact subsets of $X_{L}$ and $X_{U}$ respectively. Then the pair $\tau=\left(\tau_{L}, \tau_{U}\right)$ is said to be a rough topology on the rough set $X=\left(X_{L}, X_{U}\right)$, the pair $(X, \tau)$ is known as a rough topological space (RTS). Also in a rough topology $\tau=\left(\tau_{L}, \tau_{U}\right), \tau_{L}$ is known as the lower rough topology and $\tau_{U}$ is known as the upper rough topology on $X$.

Definition 2.11 [3]. Let $A=\left(A_{L}, A_{U}\right)$ be any rough subset of a RTS $(X, \tau)$, where $X=\left(X_{L}, X_{U}\right)$ and $\tau=\left(\tau_{L}, \tau_{U}\right)$. Then $A$ is said to be lower rough open (LRO) if the lower approximation of $A$ is in the lower rough topology. That is $A_{L} \in \tau_{L}$. Also $A$ is said to be upper rough open (URO) if the upper approximation of $A$ is in the upper rough topology. That is $A_{U} \in \tau_{U}$. $A$ is said to be rough open (RO) if and only if $A$ is lower rough open (LRO) and upper rough open (URO). That is $A=\left(A_{L}, A_{U}\right)$ rough open with respect to the RTS $(X, \tau)$ if and only if $A_{L} \in \tau_{L}$ and $A_{U} \in \tau_{U}$.

Theorem 2.12 [3]. Consider a RTS $(X, \tau)$, where $X=\left(X_{L}, X_{U}\right)$ and $\tau=\left(\tau_{L}, \tau_{U}\right)$. Let $\tau$ be the collection of all rough open subsets of $(X, \tau)$. Then $\tau$ is a topology on $X$.

Definition 2.13 [3]. If $A=\left(A_{L}, A_{U}\right)$ is any sub rough set of a rough set $X=$ $\left(X_{L}, X_{U}\right)$, then $A^{C_{L}}=X_{L} \backslash A_{L}$ is called the lower complement of $A$ and $A^{C_{U}}=X_{U} \backslash A_{U}$ is called the upper complement of $A$.

Definition 2.14 [3]. A subset $B=\left(B_{L}, B_{U}\right)$ of the RTS $(X, \tau)$, where $X=\left(X_{L}, X_{U}\right)$ and $\tau=\left(\tau_{L}, \tau_{U}\right)$ is said to be lower rough closed (LRC) if $B^{C_{L}}=X_{L} \backslash B_{L} \in \tau_{L}$. Also $B$ is said to be upper rough closed (URC) if $B^{C_{U}}=X_{U} \backslash B_{U} \in \tau_{U}$. $B$ is said to be rough closed (RC) if it is lower rough closed (LRC) and upper rough closed (URC). That is a subset $B=\left(B_{L}, B_{U}\right)$ of the RTS $(X, \tau)$ is rough closed subset iff its lower approximation is closed with respect to the lower topology and its upper approximation is closed with respect to the upper topology of $(X, \tau)$. 


\section{Main Results}

Definition 3.1. Let $X=\left(X_{L}, X_{U}\right)$ be a rough set (RS), if $x_{L} \in X_{L}$ and $x_{U} \in X_{U}$ are fixed element of the lower approximation $X_{L}$ and upper approximation $X_{U}$ respectively, then a set

$$
x_{L U}=\left\{\left(x_{L}, x_{U}\right) \mid x_{L} \in X_{L}, x_{U} \in X_{U}\right\}
$$

is called a rough point (RP) in $X$.

Remark. Given a rough set $X=\left(X_{L}, X_{U}\right)$, if there exists fixed elements $x_{L} \in X_{L}$ and $x_{U} \in X_{U}$, then a rough point $x_{L U}$ exist in $X$.

For example, considering the Example 2.6, a rough point can be given as

$$
x_{L U}=P_{1} .
$$

Definition 3.2. Let $f: X \rightarrow Y$ be a function between two sets. We called $f$ a rough function (RF) if and only if $X=\left(X_{L}, X_{U}\right)$ and $Y=\left(Y_{L}, Y_{U}\right)$, i.e., $f$ is a function between two rough sets.

Definition 3.3. Let $X$ and $Y$ be two rough sets (RS) such that $X=\left(X_{L}, X_{U}\right), Y=$ $\left(Y_{L}, Y_{U}\right)$ and $f: X \rightarrow Y$.

(i) If $x_{L U}$ is a RP in $X$, then the image $f\left(x_{L U}\right)$ of $f$ defined by

$$
f\left(x_{L U}\right)=f\left(x_{L}, x_{U}\right)=\left(f\left(x_{L}\right), f\left(x_{U}\right)\right)
$$

is a $\mathrm{RP}$ in $Y$.

(ii) If $y_{L U}$ is a RP in $Y$, then the pre-image $f^{-1}\left(y_{L U}\right)$ of $f$ defined by

$$
f^{-1}\left(y_{L U}\right)=f^{-1}\left(y_{L}, y_{U}\right)=\left(f^{-1}\left(y_{L}\right), f^{-1}\left(y_{U}\right)\right)
$$

is a $\mathrm{RP}$ in $X$.

Lemma 3.4. Suppose $f: X \rightarrow Y$ is a rough function. If $x_{L U}$ is a $R P$ in $X$, then $f\left(x_{L U}\right)$ is a $R P$ in $Y$.

Proof. The proof follows directly from Definition 3.3.

Definition 3.5. Let $(X, \tau)$ and $(Y, \sigma)$ be two rough topological spaces (RTSs) such that $X=\left(X_{L}, X_{U}\right), Y=\left(Y_{L}, Y_{U}\right), \tau=\left(\tau_{L}, \tau_{U}\right)$ and $\sigma=\left(\sigma_{L}, \sigma_{U}\right)$. A function $f:(X, \tau)$ $\rightarrow(Y, \sigma)$ is said to be rough continuous function $\left(\mathrm{R} C_{t} \mathrm{~F}\right)$ if the pre-image of each every rough open set (ROS) in $\sigma$ is a rough open set (ROS) in $\tau$. 
Theorem 3.6. Let $(X, \tau)$ and $(Y, \sigma)$ be two rough topological spaces $(R T S s)$ such that $X=\left(X_{L}, X_{U}\right), \quad Y=\left(Y_{L}, Y_{U}\right), \quad \tau=\left(\tau_{L}, \tau_{U}\right)$ and $\sigma=\left(\sigma_{L}, \sigma_{U}\right)$. A function $f:(X, \tau)$ $\rightarrow(Y, \sigma)$ is called a rough continuous function $\left(R C_{t} F\right)$ if and only if the pre-image of each rough closed set (RCS) in $\sigma$ is a rough closed set (RCS) in $\tau$.

Proof. Let $A$ be rough closed set in $(Y, \sigma)$, since the complement of the pre-image of $A$ is the pre-image of the complement of $A$. Then

$$
X \backslash f^{-1}(A)=f^{-1}(Y \backslash A) .
$$

Therefore the result follows.

Definition 3.7. Let $(X, T)$ be an ordinary topological space. The collection

$$
\tilde{\tau}=\left\{\tau_{X_{L U}} \mid \tau_{X_{L U}} \text { is rough subset of } X \text { and } \tau_{x_{L U}} \in T\right\}
$$

is rough topology $(\mathrm{RT})$ on $X$ induced by $T$. The pair $(X, \tilde{\tau})$ is called the rough topological space induced by $(X, T)$.

Note. If $I$ is the unit interval and $\xi_{I}$ is an Euclidean subspace topology on $I$, then $\left(I, \widetilde{\xi}_{I}\right)$ denotes the RTS induced by the Euclidean space $\left(I, \xi_{I}\right)$.

Definition 3.8. Let $X=\left(X_{L}, X_{U}\right)$ and $Y=\left(Y_{L}, Y_{U}\right)$ be two rough sets such that $X$ is a rough subset of $Y$. A function $f:(X, \tau) \rightarrow(Y, \sigma)$ is said to be rough continuous function $\left(\mathrm{R} C_{t} \mathrm{~F}\right)$ if the pre-image of each every rough open set (ROS) in $\sigma$ is a rough open set (ROS) in $\tau$.

Lemma 3.9. Let $(X, \tau)$ be a RTS and $A=\left(A_{L}, A_{U}\right), B=\left(B_{L}, B_{U}\right)$ be closed subsets of $X$ such that $X=A \cup B$. Let $(Y, \sigma)$ be a RTS and $f:\left(A_{L}, A_{U}\right) \rightarrow(Y, \sigma)$ and $g$ : $\left(B_{L}, B_{U}\right) \rightarrow(Y, \sigma)$ be continuous maps. If $f\left(z_{L U}\right)=g\left(z_{L U}\right)$ for all $z_{L U} \in(A \cap B)$, then the function $h:(X, \tau) \rightarrow(Y, \sigma)$ defined by

$$
h\left(z_{L U}\right)= \begin{cases}f\left(z_{L U}\right), & z_{L U} \in A \\ g\left(z_{L U}\right), & z_{L U} \in B\end{cases}
$$

is continuous.

Proof. Clearly, $h$ is unique well-defined function. We only need to show that $h$ is rough continuous.

Let $z_{L U}$ be a RCS in $(Y, \sigma)$.

$$
\begin{aligned}
h^{-1}\left(z_{L U}\right) & =X \cap h^{-1}\left(z_{L U}\right) \\
& =(A \cup B) \cap h^{-1}\left(z_{L U}\right)
\end{aligned}
$$




$$
\begin{aligned}
& =\left(A \cap h^{-1}\left(z_{L U}\right)\right) \cup\left(B \cap h^{-1}\left(z_{L U}\right)\right) \\
& =f^{-1}\left(z_{L U}\right) \cup g^{-1}\left(z_{L U}\right)
\end{aligned}
$$

Since each $f$ and $g$ is $\mathrm{RCtF}, f^{-1}\left(z_{L U}\right)$ and $g^{-1}\left(z_{L U}\right)$ are both RC in $X$. Hence $h^{-1}\left(z_{L U}\right)$ is rough closed (RC) in $X$. Consequently, $h$ is $\mathrm{RCtF}$.

Definition 3.10. Let $(W, T)$ be a topological space and $Y=\left(Y_{L}, Y_{U}\right)$ be an RS. Let $X \subseteq Y$ and $\chi_{X_{L U}}$ be rough characteristic function of $X$. Then the RT induced by $T$ is $\tilde{\tau}=\left\{\chi_{X_{L U}} \mid X \in T\right\}$ and the pair $(W, T)$ is called the rough topological space (RTS) induced by the ordinary topological space $(W, T)$.

Note. If $I=\left(I_{L}, I_{U}\right)=[0,1]$ is a unit interval and $\xi_{I}$ is an Euclidean subspace topology on $I$, then $\left(I, \widetilde{\xi}_{I}\right)$ denotes the RTS induced by the (usual) topological space $\left(I, \xi_{I}\right)$.

Definition 3.11. Let $X=\left(X_{L}, X_{U}\right)$ and $Y=\left(Y_{L}, Y_{U}\right)$ be two rough sets such that $X$ is a rough subset of $Y$. If $\chi_{X_{L U}}$ is a rough characteristic function of $X$, then the collection $\tau_{X_{L U}}=\left\{\sigma_{X_{L U}} \cap \chi_{X_{L U}} \mid \sigma_{X_{L U}} \in \tau\right\}$ is rough topology (RT) on $X$, called a rough subspace topology and the pair $\left(X, \tau_{X_{L U}}\right)$ is called a rough topological subspace $\left(\operatorname{RTS} S_{\mathrm{b}}\right)$ of $(Y, \tau)$.

Definition 3.12. Let $(X, \tau)$ and $(Y, \sigma)$ be two rough topological spaces (RTSs) and $\left(I, \widetilde{\xi}_{I}\right)$ be a RTS induced by the Euclidean space $\left(I, \xi_{I}\right)$. Let $f, g:(X, \tau) \rightarrow(Y, \sigma)$ be any two RCFs, if there exists a rough continuous function $H:(X, \tau) \times\left(I, \xi_{I}\right) \rightarrow(Y, \sigma)$ such that $H\left(x_{L U}, 0\right)=f\left(x_{L U}\right)$ and $H\left(x_{L U}, 1\right)=g\left(x_{L U}\right)$ for all RP $x_{L U} \in X$, then $f$ is rough homotopy to $g$. Moreover, the function $H$ is the rough homotopy between $f$ and $g$, denoted by $f \simeq g$.

Definition 3.13. Let $\left(I, \widetilde{\xi}_{I}\right)$ be a RTS induced by the Euclidean space $\left(I, \xi_{I}\right)$, and $(X, \tau)$ be RTS. Let $x_{L U}$ and $y_{L U}$ be any two rough points in $X$. A rough path (RPt) $h$ in $(X, \tau)$ from $x_{L U}$ to $y_{L U}$ is a rough continuous function $h:\left(I, \widetilde{\xi}_{I}\right) \rightarrow(\mathrm{X}, \tau)$ such that $h(0)=x_{L U}$ and $h(0)=y_{L U}$. Then the RPs $h(0)$ and $h(1)$ are called the initial and terminal point of $h$.

Remark. If the initial and terminal points are equal, then we called the RPt $h$, a rough loop (RL). We denote the collection of all RLs in $(X, \tau)$ by $Z\left((X, \tau), x_{L U}\right)$. The RP $x_{L U}$ is called a rough base point of $(X, \tau)$ and $\left((X, \tau), x_{L U}\right)$ is called pointed RTS. A continuity of a map between these pointed topological spaces is guaranteed by Definition 3.3. 
Definition 3.14. Let $(X, \tau)$ be RTS and $x_{L U}, y_{L U}$ be any two rough points in $X$. A $\operatorname{RTS}(X, \tau)$ is called rough path-connected if there exists an $\operatorname{RPt} h \in(X, \tau)$ with initial and terminal points $x_{L U}$ and $y_{L U}$ respectively.

Definition 3.15. Let $h$ be a rough path (RPt) in $(X, \tau)$ having the initial and finial points as $x_{L U}$ and $y_{L U}$, respectively. The inverse of $h$ is the rough path $h^{-1}$ defined by $h^{-1}\left(z_{L U}\right)=h\left(1-z_{L U}\right)$ for all $z_{L U} \in\left(I, \widetilde{\xi}_{I}\right)$, where $y_{L U}$ and $x_{L U}$ are the initial and finial points, respectively.

Definition 3.16. Let $x_{L U}$ be a RP in $(X, \tau)$. Then a constant RPt in $(X, \tau)$ is defined as $i$ based at $x_{L U}$ such that $i\left(x_{L U}\right)=x_{L U}$.

Definition 3.17. Let $\left(I, \widetilde{\zeta}_{I}\right)$ and $\left(I, \widetilde{\zeta}_{I}\right)$ be any RTSs introduced by the Euclidean spaces $\left(I, \xi_{I}\right)$ and $\left(I, \zeta_{I}\right)$ respectively. Let $(X, \tau)$ be a RTS, two RPts $h$ and $j$ in $(X, \tau)$ (having the initial and terminal points as $x_{L U}$ and $y_{L U}$ respectively) are said to be rough path homotopy $(\mathrm{RPH})$ (i.e., $h \ddot{\sim} j)$ if there exists a rough continuous function $\left(\mathrm{R} C_{t} \mathrm{~F}\right)$ $H:\left(I, \widetilde{\xi}_{I}\right) \times\left(I, \widetilde{\zeta}_{I}\right) \rightarrow(Y, \sigma)$ such that $H\left(w_{L U}, 0\right)=h\left(w_{L U}\right)$ and $H\left(w_{L U}, 1\right)=$ $j\left(w_{L U}\right)$ for all $w_{L U} \in\left(I, \widetilde{\xi}_{I}\right)$

$$
H\left(0, z_{L U}\right)=x_{L U} \text { and } H\left(1, z_{L U}\right)=y_{L U} \text { for all } z_{L U} \in\left(I, \widetilde{\zeta}_{I}\right) .
$$

Then the function $H$ is called a rough homotopy (RH) between $h$ and $j$.

Theorem 3.18. The relation $\ddot{\sim}$ on the $\operatorname{set} Z\left((X, \tau), x_{L U}\right)$ is an equivalent relation.

Proof. To show this, it is suffices to show that the following are satisfied:

(i) Reflexive Suppose $h \in Z\left((X, \tau), x_{L U}\right)$. A function $H:\left(I, \widetilde{\zeta}_{I}\right) \times\left(I, \widetilde{\zeta}_{I}\right) \rightarrow(Y, \sigma)$ defined by $H\left(w_{L U}, z_{L U}\right)=x_{L U}$ for all $w_{L U}, z_{L U} \in I$ is RH between $h$ and $h$ i.e., $x_{L U}$ is a constant function which is continuous,

$$
\begin{aligned}
& H\left(w_{L U}, 0\right)=H\left(w_{L U}, 1\right)=x_{L U} \text { for all } w_{L U} \in\left(I, \widetilde{\zeta}_{I}\right) \\
& H\left(0, z_{L U}\right)=H\left(1, z_{L U}\right)=y_{L U} \text { for all } z_{L U} \in\left(I, \widetilde{\zeta}_{I}\right) \text { and } x_{L U} \text { is a constant function. }
\end{aligned}
$$

Thus the result follows.

(ii) Symmetry Suppose $h, j \in Z\left((X, \tau), x_{L U}\right)$ such that $h \ddot{\sim} j$ then $j \ddot{\sim} h$. Let $H$ : $\left(I, \widetilde{\zeta}_{I}\right) \times\left(I, \widetilde{\zeta}_{I}\right) \rightarrow(Y, \sigma)$ be RH between $h$ and $j$ i.e., $H: h \ddot{\sim} j$ then we have $h$ $\ddot{\sim} H^{-1}: j$. 
Suppose $K:\left(I, \widetilde{\xi}_{I}\right) \times\left(I, \widetilde{\zeta}_{I}\right) \rightarrow(Y, \sigma)$ is a RH between $j$ and $h$ i.e., $K: j \ddot{\sim} h$. Which implies $K\left(w_{L U}, z_{L U}\right)=H^{-1}\left(w_{L U}, z_{L U}\right)=H\left(w_{L U}, 1-z_{L U}\right)$. Thus the result follows.

(iii) Transitive Suppose $h, j, k \in Z\left((X, \tau), x_{L U}\right)$ such that $h \ddot{\sim} j$ and $j \ddot{\simeq} k$. Suppose the RH between $(h$ and $j)$ and $(j$ and $k)$ are $H:\left(I, \widetilde{\xi}_{I}\right) \times\left(I, \widetilde{\zeta}_{I}\right) \rightarrow(Y, \sigma)$ and $K:$ $\left(I, \widetilde{\zeta}_{I}\right) \times\left(I, \widetilde{\zeta}_{I}\right) \rightarrow(\mathrm{Y}, \sigma)$ respectively. Then a function $L:\left(I, \widetilde{\xi}_{I}\right) \times\left(I, \widetilde{\zeta}_{I}\right) \rightarrow(Y, \sigma)$ defined by

$$
L\left(w_{L U}, z_{L U}\right)=\left\{\begin{aligned}
H\left(w_{L U}, 2 z_{L U}\right), & 0 \leq z_{L U} \leq \frac{1}{2} \\
K\left(w_{L U}, 2 z_{L U}-1\right), & \frac{1}{2} \leq z_{L U} \leq 1
\end{aligned}\right.
$$

is the homotopy between $h$ and $k$ i.e. $L$ is continuous by Lemma 3.9, $L\left(w_{L U}, 0\right)=$ $H\left(w_{L U}, 0\right)=h\left(w_{L U}\right), L\left(w_{L U}, 1\right)=H\left(w_{L U}, 1\right)=k\left(w_{L U}\right)$ and $L\left(0, z_{L U}\right)=H\left(0, z_{L U}\right)=$ $K\left(1,2 z_{L U}-1\right)$. Thus $h \ddot{\sim} k$. Since $\ddot{\sim}$ on the set $Z\left((X, \tau), x_{L U}\right)$ is an equivalent relation as described above, we denote the equivalence classes determined by RL $h$ by $[h]$ and we call it the rough homotopy class of $h$ (RHC). The collection of such rough homotopy classes will be denoted by $\pi_{1}\left((X, \tau), x_{L U}\right)$.

Now we are going to define an operation between the elements of $Z\left((X, \tau), x_{L U}\right)$ which are RLs.

This will be follow by proving that the collection $\pi_{1}\left((X, \tau), x_{L U}\right)$ form a group under the operation called fundamental group (or first homotopy group) of RTS $(X, \tau)$.

Definition 3.19. Let $h, j \in Z\left((X, \tau), x_{L U}\right)$. The operation $*$ for all $h, j \in$ $Z\left((X, \tau), x_{L U}\right)$ is defined as a loop $k=h * j \in Z\left((X, \tau), x_{L U}\right)$ such that

$$
k\left(z_{L U}\right)=\left\{\begin{aligned}
h\left(2 z_{L U}\right), & 0 \leq z_{L U} \leq \frac{1}{2} \\
j\left(2 z_{L U}-1\right), & \frac{1}{2} \leq z_{L U} \leq 1
\end{aligned}\right.
$$

where $h(1)=j(0)$ for all $z_{L U} \in\left(I, \widetilde{\xi}_{I}\right)$.

Definition 3.20. Let $[h],[j] \in \pi_{1}\left((X, \tau), x_{L U}\right)$, the product and the inverse of $[h]$ and $[j]$ is defined by $[h] \cdot[j]=[h * j]$ and $[h]^{-1}=\left[h^{-1}\right]$ respectively.

Lemma 3.21. The operation $\cdot$ on $\pi_{1}\left((X, \tau), x_{L U}\right)$ is closed. 
Proof. Let $[h],[j] \in \pi_{1}\left((\mathrm{X}, \tau), x_{L U}\right)$.

Goal: The goal is to show that there is $[k] \in \pi_{1}\left((X, \tau), x_{L U}\right)$ such that $[h] \cdot[j]=[k]$.

To show this it suffices to show that $k \ddot{\sim} h * j$.

Consider the function $k$ define as

$$
k\left(w_{L U}\right)= \begin{cases}h(0), & w_{L U}=0 \\ j(0), & 0<w_{L U} \leq 1\end{cases}
$$

and the concatenation of $h$ and $j$ define as

$$
h * j\left(w_{L U}\right)=\left\{\begin{aligned}
h\left(2 w_{L U}\right), & 0 \leq w_{L U} \leq \frac{1}{2} \\
j\left(2 w_{L U}-1\right), & \frac{1}{2} \leq w_{L U} \leq 1
\end{aligned}\right.
$$

where $h(1)=j(0)$. Then the function $H:\left(I, \widetilde{\xi}_{I}\right) \times\left(I, \widetilde{\zeta}_{I}\right) \rightarrow(Y, \sigma)$ defined by

$$
H\left(w_{L U}, z_{L U}\right)=\left\{\begin{aligned}
h\left(\left(2 w_{L U}\right)\left(1-z_{L U}\right),\right. & 0 \leq w_{L U} \leq \frac{1-z_{L U}}{2} \\
j\left(\left(2 w_{L U}-1\right)\left(1-z_{L U}\right)\right), & \frac{1-z_{L U}}{2} \leq w_{L U} \leq 1
\end{aligned}\right.
$$

is homotopy between $k$ and $(h * j)$ i.e., $H$ is continuous by Lemma 3.9,

$$
\begin{aligned}
& H\left(w_{L U}, 0\right)=\left\{\begin{aligned}
h\left(2 w_{L U}\right), & 0 \leq w_{L U} \leq \frac{1}{2} \\
j\left(2 w_{L U}-1\right), & \frac{1}{2} \leq w_{L U} \leq 1
\end{aligned}\right. \\
& H\left(w_{L U}, 1\right)=k\left(2 w_{L U}\right) \text { and } H\left(0, z_{L U}\right)=j(0), H\left(1-z_{L U}\right)=h(1) \text {. }
\end{aligned}
$$

Therefore $k \ddot{\sim}(h * j)$ for all $h, j, k \in Z\left((X, \tau), x_{L U}\right)$. Thus, the result follows.

Lemma 3.22. The operation $\cdot$ on $\pi_{1}\left((X, \tau), x_{L U}\right)$ is associative.

Proof. Let $[h],[j],[k] \in \pi_{1}\left((X, \tau), x_{L U}\right)$.

Goal: The goal is to show that $([h] \cdot[j]) \cdot[k]=[h] \cdot([j] \cdot[k])$.

To show this, it is suffices to show that $(h * j) * k \ddot{\sim} h *(j * k)$ for all $h, j, k \in$ $Z\left((X, \tau), x_{L U}\right)$.

Since $[(h * j) * k]=([h] \cdot[j]) \cdot[k]$ and $[h *(j * k)]=[h] \cdot([j] \cdot[k])$, consider 


$$
(h * j) * k\left(z_{L U}\right)=\left\{\begin{aligned}
h\left(4 z_{L U}\right), & 0 \leq z_{L U} \leq \frac{1}{4} \\
j\left(4 z_{L U}-1\right), & \frac{1}{4} \leq z_{L U} \leq \frac{1}{2} \\
k\left(2 z_{L U}-1\right), & \frac{1}{2} \leq z_{L U} \leq 1
\end{aligned}\right.
$$

where $h(1)=j(0)$ and $j(1)=k(0)$ for all $z_{L U} \in\left(I, \widetilde{\xi}_{I}\right)$

and

$$
h *(j * k)\left(z_{L U}\right)=\left\{\begin{array}{r}
h\left(2 z_{L U}\right), 0 \leq z_{L U} \leq \frac{1}{2} \\
j\left(4 z_{L U}-2\right), \frac{1}{2} \leq z_{L U} \leq \frac{3}{4} \\
k\left(4 z_{L U}-3\right), \frac{3}{4} \leq z_{L U} \leq 1
\end{array}\right.
$$

where $h(1)=j(0)$ and $j(1)=k(0)$ for all $z_{L U} \in\left(I, \widetilde{\xi}_{I}\right)$.

Then we define the homotopy between $(h * j) * k$ and $h *(j * k)$ as

$$
\begin{aligned}
& H\left(z_{L U}, w_{L U}\right) \\
= & \begin{cases}h\left(\left(4 z_{L U}\right) w_{L U}+\left(2 z_{L U}\right)\left(1-w_{L U}\right)\right), & 0 \leq z_{L U} \leq\left(\frac{w_{L U}}{4}+\frac{1-w_{L U}}{2}\right) \\
j\left(\left(4 z_{L U}-1\right) w_{L U}+\left(4 z_{L U}-2\right)\left(1-w_{L U}\right)\right), & \left(\frac{w_{L U}}{4}+\frac{1-w_{L U}}{2}\right) \leq z_{L U} \leq\left(\frac{w_{L U}}{2}+\frac{3\left(1-w_{L U}\right)}{4}\right) \\
k\left(\left(2 z_{L U}-1\right) w_{L U}+\left(4 z_{L U}-3\right)\left(1-w_{L U}\right)\right), & \left(\frac{w_{L U}}{2}+\frac{3\left(1-w_{L U}\right)}{4}\right) \leq z_{L U} \leq 1\end{cases}
\end{aligned}
$$

which implies that

$$
\begin{gathered}
H\left(z_{L U}, 0\right)=\left\{\begin{aligned}
h\left(2 z_{L U}\right), & 0 \leq z_{L U} \leq \frac{1}{2} \\
j\left(4 z_{L U}-2\right), & \frac{1}{2} \leq z_{L U} \leq \frac{3}{4} \\
k\left(4 z_{L U}-3\right), & \frac{3}{4} \leq z_{L U} \leq 1
\end{aligned}\right. \\
H\left(z_{L U}, 1\right)=\left\{\begin{array}{cc}
h\left(4 z_{L U}\right), & 0 \leq z_{L U} \leq \frac{1}{4} \\
j\left(4 z_{L U}-1\right), & \frac{1}{4} \leq z_{L U} \leq \frac{1}{2} \\
k\left(2 z_{L U}-1\right), & \frac{1}{2} \leq z_{L U} \leq 1
\end{array}\right.
\end{gathered}
$$

Clearly, $H$ is continuous by Lemma 3.9, since $H\left(z_{L U}, 0\right)=h *(j * k)\left(z_{L U}\right)$,

$$
H\left(z_{L U}, 1\right)=(h * j) * k\left(z_{L U}\right), H\left(0, w_{L U}\right)=h(0) \text { and } H\left(1, w_{L U}\right)=k(1)
$$

then $(h * j) * k \ddot{\sim} h *(j * k)$. Thus, the result follows. 
Lemma 3.23. Let $i:\left(I, \widetilde{\zeta}_{I}\right) \rightarrow\left(I, \widetilde{\zeta}_{I}\right)$ be a constant loop. Then $[i]$ is the identity of $\pi_{1}\left((X, \tau), x_{L U}\right)$.

Proof. Let $i\left(z_{L U}\right)=h(1)$ for all $z_{L U} \in\left(I, \widetilde{\xi}_{I}\right)$.

Goal: We are to show that there exists an identity element $[i] \in \pi_{1}\left((X, \tau), x_{L U}\right)$ such that $[i] \cdot[h]=[h]=[h] \cdot[i]$ for all $[h] \in \pi_{1}\left((X, \tau), x_{L U}\right)$.

To show this, it is suffices to show that:

(i) $h \ddot{\simeq}(i * h) \quad$ (ii) $h \ddot{\sim}(h * i)$

(i) Consider the concatenation of a constant loop $i:\left(I, \widetilde{\xi}_{I}\right) \rightarrow\left(I, \widetilde{\zeta}_{I}\right)$ and a loop $h$

$$
i * h\left(z_{L U}\right)= \begin{cases}i\left(2 z_{L U}\right), & 0 \leq z_{L U} \leq \frac{1}{2} \\ h\left(2 z_{L U}-1\right), & \frac{1}{2} \leq z_{L U} \leq 1 .\end{cases}
$$

Thus, the function $H:\left(I, \widetilde{\zeta}_{I}\right) \times\left(I, \widetilde{\zeta}_{I}\right) \rightarrow(Y, \sigma)$ defined by

$$
H\left(z_{L U}, w_{L U}\right)= \begin{cases}i\left(z_{L U}\right), & 0 \leq z_{L U} \leq \frac{1-w_{L U}}{2} \\ h\left(\left(z_{L U}\right)\left(w_{L U}\right)+\left(2 z_{L U}-1\right)\left(1-w_{L U}\right)\right), & \frac{1-w_{L U}}{2} \leq z_{L U} \leq 1\end{cases}
$$

is homotopy between $h$ and $(i * h)$ i.e., $H$ is continuous by Lemma 3.7,

$$
H\left(z_{L U}, 0\right)= \begin{cases}i\left(2 z_{L U}\right), & 0 \leq z_{L U} \leq \frac{1}{2} \\ h\left(2 z_{L U}-1\right), & \frac{1}{2} \leq z_{L U} \leq 1\end{cases}
$$

$H\left(z_{L U}, 1\right)=h\left(z_{L U}\right), H\left(0, w_{L U}\right)=i(0)$ and $H\left(1, w_{L U}\right)=h(1)$. Then $h \ddot{\sim}(i * h)$.

(ii) Consider the concatenation of a loop $h$ and a constant loop $i:\left(I, \widetilde{\zeta}_{I}\right) \rightarrow\left(I, \widetilde{\zeta}_{I}\right)$

$$
h * i\left(z_{L U}\right)= \begin{cases}h\left(2 z_{L U}\right), & 0 \leq z_{L U} \leq \frac{1}{2} \\ i\left(z_{L U}\right), & \frac{1}{2} \leq z_{L U} \leq 1\end{cases}
$$

Thus, the function $H:\left(I, \widetilde{\xi}_{I}\right) \times\left(I, \widetilde{\zeta}_{I}\right) \rightarrow(Y, \sigma)$ defined by

$$
K\left(z_{L U}, w_{L U}\right)= \begin{cases}h\left(\left(z_{L U}\right)\left(w_{L U}\right)+\left(2 z_{L U}\right)\left(1-w_{L U}\right)\right), & 0 \leq z_{L U} \leq \frac{1+w_{L U}}{2} \\ i\left(z_{L U}\right), & \frac{1+w_{L U}}{2} \leq z_{L U} \leq 1\end{cases}
$$

is homotopy between $h$ and $(h * i)$ i.e., $H$ is continuous by Lemma 3.9, 


$$
K\left(z_{L U}, 0\right)= \begin{cases}h\left(2 z_{L U}\right), & 0 \leq z_{L U} \leq \frac{1}{2} \\ i\left(z_{L U}\right), & \frac{1}{2} \leq z_{L U} \leq 1\end{cases}
$$

$K\left(z_{L U}, 1\right)=h\left(z_{L U}\right), K\left(0, w_{L U}\right)=h(0)$ and $K\left(1, w_{L U}\right)=i(1)$. Then $h \ddot{\sim}(i * h)$.

Hence, $[i] \cdot[h]=[h]=[h] \cdot[i]$ for all $[h] \in \pi_{1}\left((X, \tau), x_{L U}\right)$. Thus, the result follows.

Lemma 3.24. For each $[h] \in \pi_{1}\left((X, \tau), x_{L U}\right)$ there exists $\left[h^{-1}\right] \in \pi_{1}\left((X, \tau), x_{L U}\right)$ such that

$$
[h] \cdot\left[h^{-1}\right]=[i]=\left[h^{-1}\right] \cdot[h] .
$$

Proof. Let $[h] \in \pi_{1}\left((X, \tau), x_{L U}\right)$.

Goal: We will show that there exists $\left[h^{-1}\right] \in \pi_{1}\left((X, \tau), x_{L U}\right)$ such that

$$
[h] \cdot\left[h^{-1}\right]=[i]=\left[h^{-1}\right] \cdot[h] .
$$

To prove this, it is suffices to show there exists a constant loop $i:\left(I, \widetilde{\xi}_{I}\right) \rightarrow\left(I, \widetilde{\zeta}_{I}\right)$ such that $h * h^{-1} \ddot{\sim} i \ddot{\sim} h^{-1} * h$ i.e.

(i) $i \ddot{\sim}\left(h * h^{-1}\right)$

(ii) $i \ddot{\sim}\left(h^{-1} * h\right)$

For (i): We define a concatenation

$$
h * h^{-1}\left(z_{L U}\right)=\left\{\begin{array}{cc}
h\left(2 z_{L U}\right), & 0 \leq z_{L U} \leq \frac{1}{2} \\
h^{-1}\left(2 z_{L U}-1\right), & \frac{1}{2} \leq z_{L U} \leq 1
\end{array}\right.
$$

such that $h(1)=h^{-1}(0)$, then the function $H:\left(I, \widetilde{\xi}_{I}\right) \times\left(I, \widetilde{\zeta}_{I}\right) \rightarrow(Y, \sigma)$ defined by

$$
H\left(z_{L U}, w_{L U}\right)= \begin{cases}h\left(2 z_{L U}\right)\left(1-w_{L U}\right) & 0 \leq z_{L U} \leq \frac{1-w_{L U}}{2} \\ h^{-1}\left(\left(2 z_{L U}-1\right)\left(1-w_{L U}\right)\right) & \frac{1-w_{L U}}{2} \leq z_{L U} \leq 1\end{cases}
$$

is homotopy between $i$ and $\left(h * h^{-1}\right)$, i.e., $H$ is continuous by Lemma 3.9,

$$
H\left(z_{L U}, 0\right)= \begin{cases}i\left(2 z_{L U}\right), & 0 \leq z_{L U} \leq \frac{1}{2} \\ h\left(2 z_{L U}-1\right), & \frac{1}{2} \leq z_{L U} \leq 1\end{cases}
$$

$H\left(z_{L U}, 1\right)=i\left(z_{L U}\right), H\left(0, w_{L U}\right)=h(0)$ and $H\left(1, w_{L U}\right)=h^{-1}\left(1-w_{L U}\right)$. Then $i \ddot{\sim}(h *$ $\left.h^{-1}\right)$. 
(ii) Considering the concatenation

$$
h^{-1} * h\left(z_{L U}\right)= \begin{cases}h^{-1}\left(2 z_{L U}\right), & 0 \leq z_{L U} \leq \frac{1}{2} \\ h\left(2 z_{L U}-1\right), & \frac{1}{2} \leq z_{L U} \leq 1\end{cases}
$$

such that the function $K:\left(I, \widetilde{\xi}_{I}\right) \times\left(I, \widetilde{\zeta}_{I}\right) \rightarrow(Y, \sigma)$ defined by

$$
K\left(z_{L U}, w_{L U}\right)= \begin{cases}h^{-1}\left(2 z_{L U}\right)\left(1-w_{L U}\right) & 0 \leq z_{L U} \leq \frac{1+w_{L U}}{2} \\ h\left(\left(2 z_{L U}-1\right)\left(1-w_{L U}\right)\right) & \frac{1+w_{L U}}{2} \leq z_{L U} \leq 1\end{cases}
$$

is homotopy between $i$ and $\left(h^{-1} * h\right)$ i.e., $K$ is continuous by Lemma 3.9,

$$
K\left(z_{L U}, 0\right)= \begin{cases}h^{-1}\left(2 z_{L U}\right), & 0 \leq z_{L U} \leq \frac{1}{2} \\ h\left(2 z_{L U}-1\right), & \frac{1}{2} \leq z_{L U} \leq 1\end{cases}
$$

$K\left(z_{L U}, 1\right)=i\left(z_{L U}\right), \quad K\left(0, w_{L U}\right)=h^{-1}(0)$ and $K\left(1, w_{L U}\right)=h(1)$. Then $i \ddot{\sim}\left(h^{-1} * h\right)$.

Hence

$$
[h] \cdot\left[h^{-1}\right]=[i]=\left[h^{-1}\right] \cdot[h] .
$$

Thus, the result follows.

Corollary 3.25. The set $\pi_{1}\left((X, \tau), x_{L U}\right)$ together with the operation $\cdot$ as defined above is a group.

Proof. The prove follows immediately from Lemmas 3.21, 3.22, 3.23 and 3.24.

Proposition 3.26. Let $\beta:\left((X, \tau), x_{L U}\right) \rightarrow\left((Y, \tau), y_{L U}\right), \sigma:\left((Y, \tau), y_{L U}\right) \rightarrow\left((Z, \tau), z_{L U}\right)$ be two continuous maps of pointed rough topological spaces. Then,

a) $\beta$ induces a homomorphism $\pi_{1}(\beta): \pi_{1}\left((X, \tau), x_{L U}\right) \rightarrow \pi_{1}\left((Y, \tau), y_{L U}\right)$

b) $\pi_{1}\left((X, \tau), x_{L U}\right) \ddot{\check{ }} \pi_{1}\left((Y, \tau), y_{L U}\right)$

c) The homomorphism $\pi_{1}(\sigma) \cdot \pi_{1}(\beta): \pi_{1}\left((X, \tau), x_{L U}\right) \rightarrow \pi_{1}\left((Z, \tau), z_{L U}\right)$ is equal to the homomorphism $\pi_{1}(\sigma * \beta): \pi_{1}\left((X, \tau), x_{L U}\right) \rightarrow \pi_{1}\left((Z, \tau), z_{L U}\right)$

d) For $\iota:\left((X, \tau), x_{L U}\right) \rightarrow\left((X, \tau), x_{L U}\right)$ the identity map, we have $\pi_{1}(\iota): \pi_{1}\left((X, \tau), x_{L U}\right)$ $\rightarrow \pi_{1}\left((X, \tau), x_{L U}\right)$ as an identity map of groups which is isomorphism.

\section{Proof.}

For (a): Define $\pi_{1}(\beta): \pi_{1}\left((X, \tau), x_{L U}\right) \rightarrow \pi_{1}\left((Y, \tau), y_{L U}\right)$ by $\pi_{1}(\beta)([h])=[\beta * h]$ for all $[h] \in \pi_{1}\left((X, \tau), x_{L U}\right)$. Let $[h],[j] \in \pi_{1}\left((X, \tau), x_{L U}\right)$. Then 


$$
\begin{aligned}
\pi_{1}(\beta)([h]) \cdot \pi_{1}(\beta)([j]) & =[\beta * h] \cdot[\beta * j] \\
& =[(\beta * h) *(\beta * j)] \\
& =[\beta *(h * j)] \\
& =\pi_{1}(\beta)([h * j]) \\
& =\pi_{1}(\beta)([h] \cdot[j]) .
\end{aligned}
$$

For (b): It is suffices to show that $\pi_{1}(\beta): \pi_{1}\left((X, \tau), x_{L U}\right) \rightarrow \pi_{1}\left((Y, \tau), y_{L U}\right)$ is bijective.

Let $j^{-1}$ be rough path between point $y_{L U}$ and $x_{L U}$, define $\pi_{1}(\alpha): \pi_{1}\left((Y, \tau), y_{L U}\right) \rightarrow$ $\pi_{1}\left((X, \tau), x_{L U}\right)$ by $\pi_{1}(\alpha)([j])=\left[\beta^{-1} * j\right]$, then

$$
\begin{aligned}
\pi_{1}(\alpha \beta)([j]) & =\pi_{1}(\alpha) \pi_{1}(\beta)([j]) \\
& =\pi_{1}(\alpha)([\beta * j]) \\
& =\left[\beta^{-1} * \beta * j\right] \\
& =[\iota * j] \\
& =[j] .
\end{aligned}
$$

Also,

$$
\begin{aligned}
\pi_{1}(\beta \alpha)([j]) & =\pi_{1}(\beta) \pi_{1}(\alpha)([j]) \\
& =\pi_{1}(\beta)([\alpha * j]) \\
& =\left[\beta * \beta^{-1} * j\right] \\
& =[\iota * j] \\
& =[j] .
\end{aligned}
$$

For (c): For all $[h] \in \pi_{1}\left((X, \tau), x_{L U}\right)$, we have

$$
\begin{aligned}
\pi_{1}(\sigma) \cdot \pi_{1}(\beta)([h]) & =\pi_{1}(\sigma)([\beta * h]) \\
& =[\sigma *(\beta * h)] \\
& =[(\sigma * \beta) * h] \\
& =\pi_{1}(\sigma * \beta)[h] .
\end{aligned}
$$

For (d): It is suffices to note that $\pi_{1}(\iota)$ sends any rough loop to itself, since $\iota * h=h$ 
for all $\mathrm{RL} h \in Z\left((X, \tau), x_{L U}\right)$. Therefore it induces the identity morphism on fundamental groups of RTS.

Follow from (c) above $\beta * \beta^{-1}=\iota$ and $\beta^{-1} * \beta=\iota$ are rough identity functions. Therefore $\pi_{1}(\beta) \cdot \pi_{1}\left(\beta^{-1}\right)=\pi_{1}(\iota)$ and $\pi_{1}\left(\beta^{-1}\right) \cdot \pi_{1}(\beta)=\pi_{1}(\iota)$ are identity isomorphism, hence $\pi_{1}(\beta)$ is an isomorphism.

\section{Conclusion}

We considered the theory of rough sets to the structure of fundamental group. We introduced the concept of fundamental group of rough topological spaces by defining the concept rough path, rough loop and rough homotopy using the definition of rough topological spaces in [3].

\section{References}

[1] A. Hatcher, Algebraic Topology, Cambridge University Press, Cambridge, 2002.

[2] H. Poincaré, Analysis situs and its five supplements, Papers on Topology 2 (2009), 2228.

[3] B.P. Mathew and S.J. John, On rough topological spaces, International Journal of Mathematical Archive 3(9) (2012), 3413-3421.

[4] B.P. Mathew and S.J. John, I-rough topological spaces, International Journal of Rough Sets and Data 3(1) (2016), 98-311. https://doi.org/10.4018/IJRSDA.2016010106

[5] B.P. Mathew and S.J. John, Some special properties of I-rough topological spaces, Annals of Pure and Applied Mathematical 12(2) (2016), 111-122.

[6] B.P. Mathew and S.J. John, Some studies on I-rough topological spaces, International Journal of Mathematical Archive 11(3) (2020), 20-29.

[7] E.A. Az-zo'bi, M.F. Marashdah and R.F. Uzabashy, The fundamental group of intuitionistic fuzzy topological spaces, Applied Mathematical Sciences 8(157) (2014), 7829-7843. https://doi.org/10.12988/ams.2014.49719

[8] E.F. Lashin, A.M. Kozae, A.A. Abo Khadra and T. Medhat, Rough set theory for topological spaces, Internat. J. Approx. Reason. 40 (2005), 35-43.

https://doi.org/10.1016/j.ijar.2004.11.007

[9] G. Varma and S.J. John, Generalized multi-fuzzy rough sets and the induced topology, IOSR Journal of Mathematics (IOSR-JM) (2017), 2278-5728.

[10] G. Zhang, On topological structures of IVF approximation spaces, Fuzzy Inf. Eng. 8 (2016), 217-227. https://doi.org/10.1016/j.fiae.2016.06.006 
[11] H. Zhang, L. Xiong and W. Ma, Generalized intuitionistic fuzzy soft rough set and its application in decision making, J. Comput. Anal. Appl. 20(4) (2016), 750-766.

[12] J. Brazas, The fundamental group as a topological group, Topology Appl. 160 (2013), 170-188. https://doi.org/10.1016/j.topol.2012.10.015

[13] J.S. Calcut, R.E. Gompf and J.D. McCarthy, On fundamental groups of quotient spaces, Topology Appl. 159 (2012), 322-330. https://doi.org/10.1016/j.topol.2011.09.038

[14] K. Saito and T. Ishibe, Monoids in the fundamental groups of the complement of logarithmic free divisors in $C^{3}$, J. Algebra 344 (2011), 137-160. https://doi.org/10.1016/j.jalgebra.2011.07.018

[15] L. Abdullateef, On algebraic properties of fundamental group of intuitionistic fuzzy topological spaces (IFTSs), Open Journal of Mathematical Sciences 4 (2020), 34-47. https://doi.org/10.30538/oms2020.0092

[16] N. Xie, On topological properties of IF approximation spaces, Fuzzy Inf. Eng. 8 (2015), 183-193. https://doi.org/10.1016/j.fiae.2015.05.004

[17] R. Mareay, Generalized rough sets based on neighborhood systems and topological spaces, J. Egyptian Math. Soc. 24 (2016), 603-608.

https://doi.org/10.1016/j.joems.2016.02.002

[18] V. Madhuri and B. Amudhambigai, The fuzzy $\mathfrak{J}^{*}$-fundamental group of fuzzy $\mathfrak{J}^{*}$ structure spaces, Int. Journal of Computational and Appl. Math. 12(1) (2017), 40-53.

[19] W. Tang, J. Wu and D. Zheng, On fuzzy rough sets and their topological structures, Math. Probl. Eng. 2014, Art. ID 546372, 17 pp. https://doi.org/10.1155/2014/546372

[20] Y.B. Jun, K.J. Lee and C.H. Park, Soft set theory applied to ideals in $d$-algebras, Comput. Math. Appl. 57 (2009), 367-378.

[21] Z. Li and R. Cui, On topological structure of intuitionistic fuzzy sets, Fuzzy Math. Inform. 5(1) (2013), 229-239.

[22] Z. Li, T. Xie and Q. Li, Topological structure of generalized rough sets, Comput. Math. Appl. 63 (2012), 1066-1071. https://doi.org/10.1016/j.camwa.2011.12.011

[23] Z. Pawalak, Rough sets, Internat. J. Comput. Inform. Sci. 11 (1982), 341-356. https://doi.org/10.1007/BF01001956

This is an open access article distributed under the terms of the Creative Commons Attribution License (http://creativecommons.org/licenses/by/4.0/), which permits unrestricted, use, distribution and reproduction in any medium, or format for any purpose, even commercially provided the work is properly cited. 